MINI-SYMPOSIUM

\title{
Role of myocardial contrast echocardiography in the clinical evaluation of acute myocardial infarction
}

\section{R Senior}

$\mathrm{T}$ he ultimate goal of treatment in acute myocardial infarction (AMI) is to salvage as much myocardium as possible with the least possible risk to the patient. In the immediate aftermath of reperfusion therapy, the clinician must determine whether the infarct related artery (IRA) is patent and if so whether successful myocardial reperfusion has been achieved. Addressing these questions expeditiously is important for subsequent treatment strategies-that is, if thrombolytic therapy has failed then the patient may be transferred for rescue coronary intervention. Furthermore, even when the patency of the IRA is restored, one has to determine whether microvascular perfusion is present. It is also important to identify the presence and extent of residual myocardial viability (MV) following AMI because subsequent revascularisation may not benefit patients with predominant myocardial necrosis, while those patients with significant MV are likely to benefit from revascularisation.

\section{MYOCARDIAL CONTRAST ECHOCARDIOGRAPHY}

Myocardial contrast echocardiography (MCE) is a technique that utilises microbubbles which remain entirely within the intravascular space and denotes the status of microvascular perfusion within that region. ${ }^{1}$ The myocardial signal assessed visually as contrast intensity reflects capillary blood volume. ${ }^{2}$ Furthermore, following destruction of microbubbles in the myocardium during high power imaging, the rate of replenishment of the myocardium reflects microbubble or myocardial blood velocity $(\beta)$. Myocardial perfusion is defined as tissue blood flow at the capillary level. The two components of tissue blood flow-capillary blood volume and blood velocity - can be assessed by MCE. The product of these two components denotes myocardial blood flow (MBF) at the tissue level. ${ }^{2}$ Thus, MCE can detect not only capillary blood volume but by virtue of its temporal resolution can also assess MBF.

\section{PATHOPHYSIOLOGY OF AMI AND RELEVANCE TO MCE}

The extent of myocardial necrosis after AMI is directly related to: (1) total duration of coronary occlusion; (2) the extent of myocardium subtended by the occluded artery; and (3) the quality of collateral circulation. Thus, following AMI the progression of myocardial necrosis may be halted if the IRA opens either spontaneously or after reperfusion therapy, or if there is sufficient collateral circulation supplying the jeopardised region despite occluded artery. Prolonged ischemia may result in the failure to establish microvascular reperfusion (low reflow or no reflow) despite restoration of epicardial coronary patency. ${ }^{3}$ No reflow is a marker of myocyte necrosis and hence residual myocardial viability. ${ }^{3}$ However, in the immediate reperfusion period coronary hyperaemia may occur and this may result in underestimation of myocardial necrosis by any technique that uses intravascular tracers like MCE. ${ }^{4}$ Finally, magnitude and spatial extent of the no reflow phenomenon varies over time. ${ }^{4}$ This dynamic feature of post-ischaemic flow must be taken into account with respect to the appropriate timing and interpretation of MCE following AMI.

\section{APPLICATION OF MCE IN AMI Determination of ultimate infarct size at the time of AMI}

Patients presenting with ongoing chest pain and ST elevation in the ECG need emergent reperfusion therapy. However, there are patients who present in the emergency department in whom chest pain has resolved despite persistent ST elevation. Under these circumstances, it is important to determine whether the myocardium is at risk of necrosis regarding proceeding for emergent perfusion therapy. Coggins and colleagues found that MCE defect size late after destruction replenishment sequence corresponded to the ultimate infarct size and that MBF assessed by MCE accurately predicted collateral blood flow during acute coronary occlusion. ${ }^{5}$ Thus it may be speculated that those patients with extensive collateral MBF may not undergo emergent revascularisation as long as they are haemodynamically stable.

\section{Assessment of IRA patency}

IRA patency may not be achieved in approximately $30 \%$ of patients after thrombolysis. Clinical predictors-that is, chest pain, resolution of ST elevation and cardiac enzyme for detecting IRA patency immediately after thrombolysis-have limited accuracy. ${ }^{6}$ IRA patency can be determined with MCE on the physiologic basis described above. During acute total coronary occlusion in the absence of collateral flow, a transmural contrast perfusion defect occurs. ${ }^{7}$ After reperfusion the defect will no longer be transmural whether infarction is absent or present; the contrast defect will be smaller than that seen during occlusion as a result of regions of post-ischaemic hyperaemia, sparing of an epicardial rim of viable tissue, or both. ${ }^{7}$ Thus, if MCE is performed before and after reperfusion therapy, the IRA patency can be determined by comparing the transmural extent of the defects in each image. If the IRA patency is not restored after thrombolysis the patient may be referred urgently for rescue percutaneous coronary intervention (PCI). On the other hand, if the IRA patency is restored, one can predict the extent of myocardial necrosis. MCE performed late (three hours) not early after restoration of patency of IRA in an experimental model showed the best predictive value for ultimate infarct sizethat is, after abatement of coronary hyperaemia. ${ }^{8}$ In a study performed by our group, it was shown that MCE performed

Abbreviations: AMI, acute myocardial infarction; IRA, infarct related artery; $M B F$, myocardial blood flow; MCE, myocardial contrast echocardiography; MV, myocardial viability; $\mathrm{PCl}$, percutaneous coronary intervention; PTCA, percutaneous transluminal coronary angioplasty 

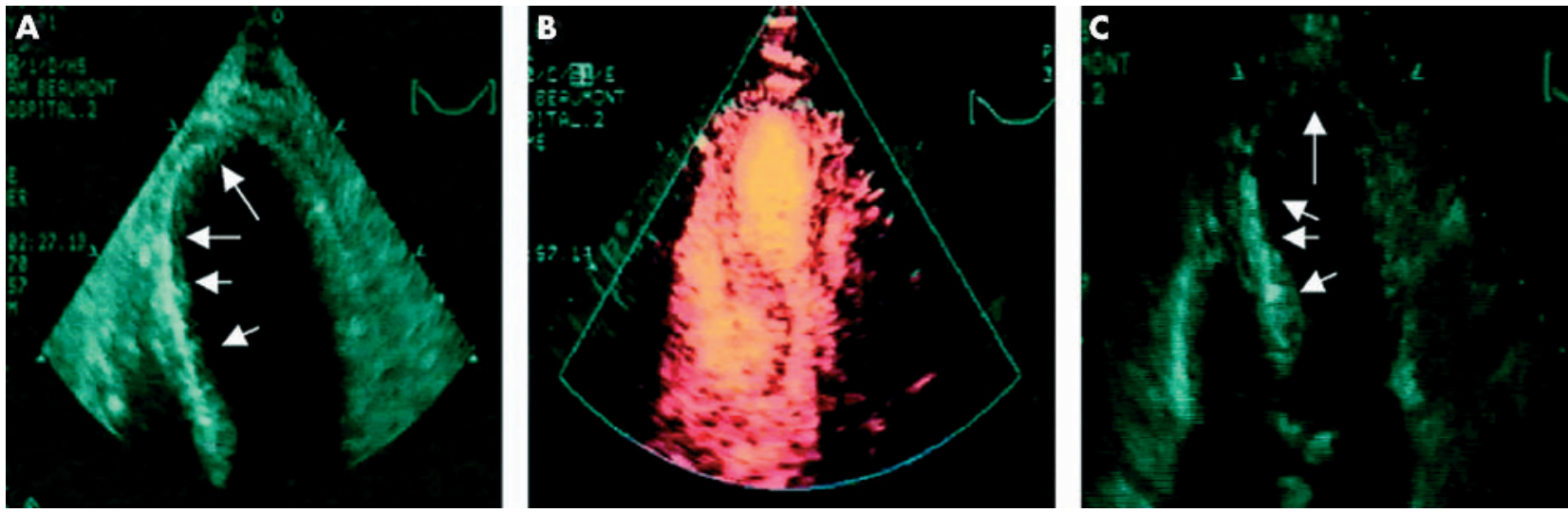

Figure 1 (A) Apical four chamber view in systole showing akinetic septum and apex (arrows) 12 hours after successful PTCA. (B) Homogeneous contrast opacification of the akinetic segments. (C) Follow up echocardiography at one month, showing recovery of function of these segments (arrows) with reduction of left ventricular end systolic value.

24 hours after restoration of IRA patency with PCI was superior to clinical and angiographic predictors of myocardial perfusion. ${ }^{\circ}$

Figure 1 shows an example of a patient with an anterior acute myocardial infarct demonstrating apico-septal akinesia 12 hours after coronary angioplasty (PTCA). However, MCE showed homogeneous opacification of the septum and apex, suggestive of preserved microvascular perfusion; this predicted recovery of function, shown by follow up echocardiography at one month. Figure 2 is an example of another patient with an anterior AMI with apico-septal akinesia 12 hours after successful PTCA. However, MCE showed no opacification of these segments, suggesting lack of microvascular perfusion despite a patent epicardial artery. The follow up echocardiogram did not show recovery of function in the akinetic segments, as predicted by MCE. ${ }^{9}$

\section{Assessment of myocardial viability after AMI}

Ragosta and colleagues noted that those with patent IRA and good contrast intensity (microvascular volume) demonstrated improvement in contractile function compared to those patients with poor contrast score one month after AMI. ${ }^{10}$ Our group similarly showed that the extent and severity of contrast defects after AMI showed a strong inverse correlation with recovery of function at three months after revascularisation. ${ }^{11}$ Ito and associates noted that in the $25 \%$ of their patient cohort with no myocardial opacification, despite a patent IRA, regional and global function were worse one month later compared with those showing opacification of the infarct bed. ${ }^{12}$ These studies established the value of intact microvasculature after AMI as assessed by MCE to predict MV.

However, MBF may be normal or reduced (patent but presence of severe flow limiting stenosis at rest of IRA, partial microembolisation of distal vessels despite patent IRA and in presence of collateral blood flow) in viable myocardium. In a study of 98 patients after AMI by Swinburn and colleagues, it was clearly shown that contrast intensity assessed early after microbubble destruction was a poor predictor of MV compared to contrast intensity assessment late after microbubble destruction. ${ }^{13}$ Indeed, because of variability in MBF in the infarct related region, assessment of contrast intensity should be continued up to 15 cardiac cycles following the destructive phase for optimum assessment of MV. ${ }^{5}$ While assessment of microvascular integrity performed as above is a reliable indicator of $\mathrm{MV}$, it may not be able to discriminate normal from minor tissue damage. Microbubble velocity $(\beta)$ and not microvascular intensity was the stronger predictor of
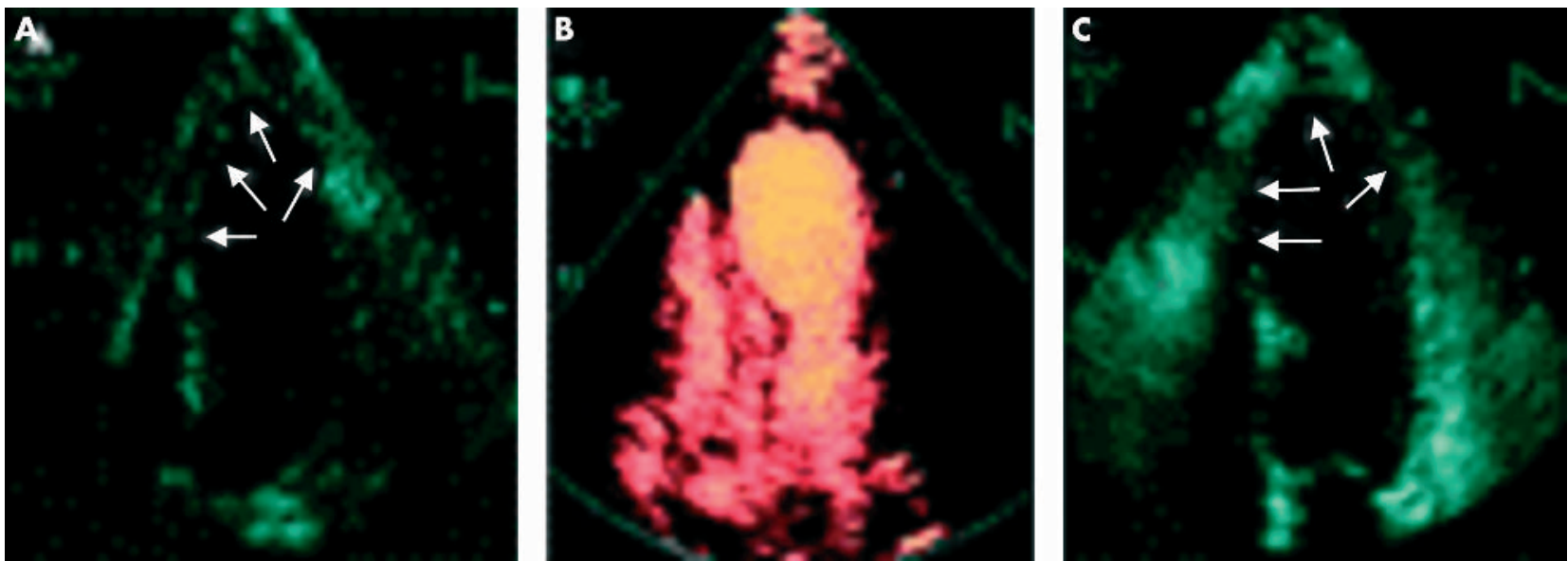

Figure 2 (A) Apical four chamber view in systole showing akinetic mid septum and apex (arrows) 12 hours after successful PTCA. (B) No contrast opacification seen in mid septum and apex. (C) Follow up echocardiography at one month, showing lack of recovery of function of these segments (arrows) with no change in left ventricular end systolic volume. 


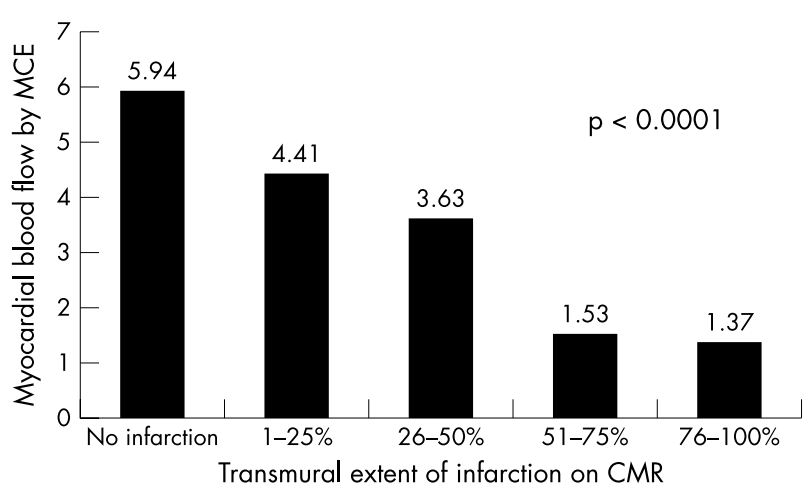

Figure 3 Quantitative myocardial contrast echocardiography predicting transmural extent of infarction.

contractile reserve in patients after AMI. ${ }^{6}{ }^{14}$ This is not surprising because $\beta$ has a stronger relation with MBF than peak contrast intensity. Contrast intensity is affected by threshholding effect and sometimes indistinguishable background noise may fail to detect differences between normal and mildly reduced microvascular volume in a small infarction. Thus, in a recent study by our group using low power MCE, infarct transmurality assessed by cardiac magnetic resonance imaging was accurately predicted by microbubble velocity and levels of $\mathrm{MBF}$ assessed by $\mathrm{MCE}^{14}$ (fig 3).

\section{Accuracy of MCE to predict myocardial viability after AMI}

Most previous studies with MCE have demonstrated high sensitivity $(75-90 \%)$ but poorer specificity $(50-60 \%)$ to identify recovery of contractile function after AMI. Most of these studies were performed early after reperfusion and assessed resting function. The combination of reactive hyperaemia, dynamic nature of changes in the microcirculation early after AMI, and the fact that AMI involving more than $20 \%$ of the subendocardium can render the myocardium akinetic despite significant epicardial and mid-MV, tend to apparently make MCE less specific for detection of MV. Technical factors like inability to distinguish microbubble signature from the underlying tissue is also important in contributing to low specificity of MCE. Recent studies, using background subtraction techniques, either on-line (low power or high power imaging - that is, power Doppler and ultraharmonics) or off-line, assessing patients 3-5 days after AMI and assessment of contractile reserve considerably improved the specificity $(80-90 \%)$ and positive predictive value $(85-90 \%)$ of MCE. ${ }^{14-16}$

\section{CONCLUSION}

The ability of MCE to image capillary blood flow makes this technique unique to assess patients with AMI which directly involves myocardial capillaries. The refinement of MCE technology today allows this technique to be used readily and reliably.

Correspondence to: Dr Roxy Senior, Northwick Park Hospital, Watford Road, Harrow, HAl 3UJ, UK; roxy.senior@virgin.net

\section{REFERENCES}

1 Kaul S, Force T. Assessment of myocardial perfusion with contrast two dimensional echocardiography. In: Weyman AE ed. Principles and practice of echocardiography, 2nd ed. Philadelphia: Lea and Febiger, 1993:687-730.

2 Wei K, Jayaweera AR, Firoozan S, et al. Quantification of myocardial blood flow with ultrasound-induced destruction of microbubbles administered as a continuous infusion. Circulation 1998;97:473-83.

3 Kloner RA, Gante CE, Jennings RB. The 'no-reflow' phenomenon after temporary coronary occlusion in the dog. J Clin Invest 1974;54:1496-508.

4 Cobb FR, Bache RJ, Rivas F, et al. Local effects of acute cellular injury on regional myocardial blood flow. J Clin Invest 1976;57:1359-68.

5 Coggins MP, Sklenar J, Le DE, et al. Noninvasive prediction of ultimate infarct size at the time of acute coronary occlusion based on the extent and magnitude of collateral-derived myocardial blood flow. Circulation 2001; 104:2471-7.

6 Califf RM, O'Neil W, Stack RS, et al. Failure of simple clinical measurements to predict perfusion status after intravenous thrombolysis. An Intern Med 1988; 108:658-62.

7 Villanueva FS, Glasheen WD, Sklenar J, et al. Assessment of risk area during coronary occlusion and infarct size after reperfusion with myocardial contrast echocardiography using left and right atrial injections of contrast. Circulation 1993;88:596-604.

8 Lafitte S, Higashiyama A, Masugata H, et al. Contrast echocardiography can assess risk area and infarct size during coronary occlusion and reperfusion: experimental validation. J Am Coll Cardiol 2002;39:1546-54.

9 Greaves K, Dixon SR, Fejka M, et al. Myocardial contrast echocardiography is superior to other known modalities for assessing myocardial perfusion after acute myocardial infarction. Heart 2003;89:139-44.

10 Ragosta M, Camarano G, Kaul S, et al. Microvascular integrity indicates myocellular viability in patients with recent myocardial infarction. New insights using myocardial contrast echocardiography. Circulation 1994;89:2562-9

11 Janardhanan R, Swinburn J, Greaves K, et al. Usefulness of myocardial contrast echocardiography using low-power continuous imaging early after acute myocardial infarction to predict late functional left ventricular recovery. Am J Cardiol 2003;92:493-7.

12 Ito $\mathbf{H}$, Tomooka T, Sakai N, et al. Lack of myocardial perfusion immediately after successful thrombolysis. A predictor of poor recovery of left ventricular function in anterior myocardial infarction. Circulation 1992;85:1699-705.

13 Swinburn JMA, Lahiri A, Senior R. Intravenous myocardial contrast echocardiography predicts recovery of dysynergic myocardium early after acute myocardial infarction. J Am Coll Cardiol 2001;38:19-25.

14 Janardhanan R, Moon JCC, Pennell DJ, et al. Prediction of the extent of myocardial necrosis and contractile reserve after reperfusion therapy following cute myocardial infarction: comparison between myocardial contrast echocardiography and contrast enhanced cardiovascular magnetic resonance. J Am Coll Cardiol 2003;1116MP-204(suppl):433A

15 Main ML, Magalski A, Chee NK, et al. Full-motion pulse inversion power Doppler contrast echocardiography differentiates stunning from necrosis and predicts recovery of left ventricular function after acute myocardial infarction. J Am Coll Cardiol 2001;38:1390-4.

16 Swinburn J, Senior R. Real-time contrast echocardiography - a new bedside technique to predict contractile reserve early after acute myocardial infarction. Eur J Echocardiogr 2002;3:95-9. 